\title{
Rapid thermal annealing of graphene-metal contact
}

Osman Balci, and Coskun Kocabas

Citation: Appl. Phys. Lett. 101, 243105 (2012);

View online: https://doi.org/10.1063/1.4769817

View Table of Contents: http://aip.scitation.org/toc/apl/101/24

Published by the American Institute of Physics

\section{Articles you may be interested in}

Contacting graphene

Applied Physics Letters 98, 053103 (2011); 10.1063/1.3549183

Improvement of graphene-metal contact resistance by introducing edge contacts at graphene under metal Applied Physics Letters 104, 183506 (2014); 10.1063/1.4875709

Contact resistivity and current flow path at metal/graphene contact Applied Physics Letters 97, 143514 (2010); 10.1063/1.3491804

Transition metal contacts to graphene Applied Physics Letters 107, 153104 (2015); 10.1063/1.4933192

Ultraviolet/ozone treatment to reduce metal-graphene contact resistance Applied Physics Letters 102, 183110 (2013); 10.1063/1.4804643

Ultra-low resistance ohmic contacts in graphene field effect transistors Applied Physics Letters 100, 203512 (2012); 10.1063/1.4719579

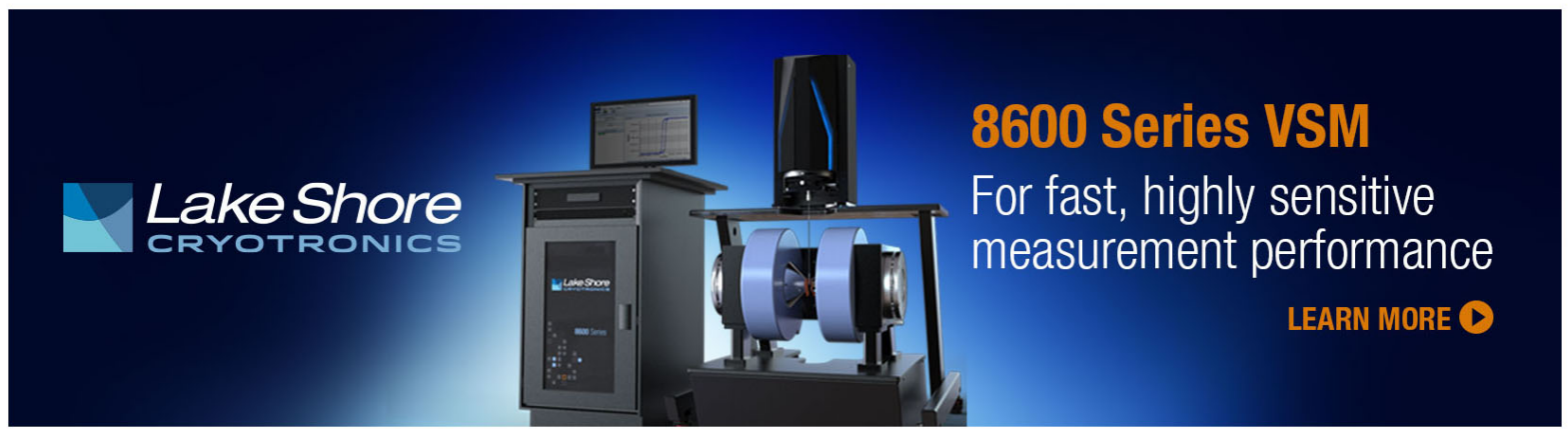




\title{
Rapid thermal annealing of graphene-metal contact
}

\author{
Osman Balci and Coskun Kocabas ${ }^{\text {a) }}$ \\ Department of Physics, Bilkent University, Ankara 06800, Turkey
}

(Received 22 July 2012; accepted 19 November 2012; published online 10 December 2012)

\begin{abstract}
High quality graphene-metal contacts are desirable for high-performance graphene based electronics. Process related factors result large variation in the contact resistance. A post-processing method is needed to improve graphene-metal contacts. In this letter, we studied rapid thermal annealing (RTA) of graphene-metal contacts. We present results of a systematic investigation of device scaling before and after RTA for various metals. The results reveal that RTA provides a convenient technique to reduce contact resistance, thus to obtain reproducible device operation. (C) 2012 American Institute of Physics. [http://dx.doi.org/10.1063/1.4769817]
\end{abstract}

Rapid thermal processes, where the processed wafer is not in thermal equilibrium with the surrounding environment, are used in semiconductor industry for a variety of purposes such as controlling doping levels, ${ }^{1}$ increasing interface qual$\mathrm{ity}^{2}$ and repairing defects. ${ }^{3}$ Rapid thermal annealing (RTA), which is classified as a subset of these processes, is commonly used to improve the quality of metal-semiconductor contacts. ${ }^{4}$ The quality of metal contacts is an important issue for graphene based electronic devices. ${ }^{5-8}$ The resistance of the graphene-metal junction, known as contact resistance, limits the performance of devices having small channel length. Particularly for high frequency electronics, ${ }^{5,9}$ which require smaller channel length for higher operation frequency, the contact resistance is the main limiting factor affecting the device operation. ${ }^{5,6}$ Graphene, which is a zerobandgap semiconducting material, shares common fabrication processes with conventional semiconducting materials; therefore, rapid thermal annealing could also be applied to improve the quality of metal contacts. In this work, we implement rapid thermal annealing of graphene based field effect transistors to reduce the contact resistance. We provide a systematic study of RTA of graphene transistors with four different contact metals, $\mathrm{Cu}, \mathrm{Ag}, \mathrm{Au}$, and Pd. We studied channel length scaling of these devices to reveal the contribution of channel and contact properties using transfer line method (TLM). The results reveal that RTA provides a convenient technique to reduce contact resistance, thus to obtain reproducible device operation.

The maximum conductance of one-dimensional conductors, such as carbon nanotubes, is defined by fundamental constants. The minimum resistance per subband is $h / e^{2}=25.8 k \Omega$, where $h$ is the Plank's constant and $e$ is the elementary charge. We can extent this formalism for a wide conductor by calculating the number of modes available on the conductor. The minimum resistance of a wide conductor with transparent contacts can be written as ${ }^{10}$

$$
R W=\frac{h}{2 e^{2}} \sqrt{\frac{\pi}{2 n}}=\frac{16.28 k \Omega}{\sqrt{n}},
$$

\footnotetext{
a) Author to whom correspondence should be addressed. Electronic mail: ckocabas@fen.bilkent.edu.tr. Tel.: +90(312)2901965.
}

where $n$ is the carrier density and $W$ is the width of the wide conductor. This minimum resistance appears to be a contact resistance for graphene transistors. Note that the contact resistance scales with the carrier density, which defines the number modes on the conductor. For graphene with a carrier density of $\sim 1 \times 10^{12} \mathrm{~cm}^{-2}$, the minimum resistance is $160 \Omega \mu \mathrm{m}$. This value is the minimum contact resistance that can be obtained with ideal transparent electrodes. Any kind of process related detrimental effects could increase the observed contact resistance. The reported values of the contact resistance of graphene-metal junction are very scattered and much larger than the expected value. This ambiguity in the contact resistance is likely related with deposition methods of metals and the quality of graphene layers. First, the adhesive layers used for electrodes (thin layer of $\mathrm{Ti}$ or $\mathrm{Cr}$ ) cause large variation in the contact resistance. Particularly, adhesive layers deposited by sputtering technique introduce structural defects on graphene, which could cause extremely large contact resistance $\left(\mathrm{R}_{\mathrm{c}}>10^{4} \Omega \mu \mathrm{m}\right){ }^{11}$ Therefore, contact metals deposited by sputtering, thermal evaporation, and e-beam evaporation provide different contact resistances. Second, the quality of graphene determines the contact resistance as well. Most of the reported experiments about contact resistance use exfoliated single layer graphene. There are a few studies on contact resistance of polycrystalline graphene synthesized by chemical vapor deposition. ${ }^{5,12,13}$

To improve the graphene-metal contacts, various techniques have been implemented. Palacios et al. ${ }^{5}$ used $\mathrm{Al}$ sacrificial layer during the lithography process to improve the surface roughness of graphene. Deposition of Al layer on graphene and removing it with tetramethylammonium hydroxide (TMAH) eliminate the organic residues and provide smooth graphene surface. They obtained contact resistance of $0.2-0.5 \mathrm{k} \Omega \mu \mathrm{m}$ for Ti:Pd:Au electrode. Watanabe et al. ${ }^{14}$ studied contact resistance of various metals $(\mathrm{Ti}, \mathrm{Ag}, \mathrm{Co}, \mathrm{Cr}$, $\mathrm{Fe}, \mathrm{Ni}$, and $\mathrm{Pd}$ ) for graphene obtained from Kish graphite. Their results show that the contact resistance is independent of work function of the contact metal. They concluded that microstructures of metals (roughness) play an important role for contact resistance. Xia et al. ${ }^{7}$ showed that carrier transport in graphene under the contact metal plays a significant role for contact resistance. Temperature dependent experiments demonstrated that a good graphene-metal contact can 
be achieved when the mean free path is larger than the metal-graphene coupling length. A contact resistance of $170 \Omega \mu \mathrm{m}$ for Pd (without adhesive layer) at room temperature was reported. They also provided a theoretical explanation of the carrier transport at graphene-metal junction. Franklin et al. ${ }^{13}$ introduced the concept of double contacts to reduce the contact resistance. They used metals on top and bottom of graphene and obtained contact resistance of $320 \Omega \mu \mathrm{m}$. Very recently Song et al. ${ }^{15}$ measured the work function of graphene under the metal electrodes using capacitance-voltage measurement. They observed that the work function of graphene is affected by the metal and pinned to the work function of electrodes.

We can draw four main conclusions from the previous results; (1) the contact resistance is independent of the work function of the contact metal, (2) roughness of the metal plays a significant role in the contact resistance, (3) residues on graphene increase the contact resistance, and (4) the metal contacts dope the graphene depending on difference of the work functions. All these process-dependent factors result large variation in the contact resistance. Thus, a postprocessing method is desirable to improve graphene-metal contacts.

Fig. 1(a) shows a schematic drawing of a back-gated graphene transistor used in this study. Graphene layers were grown on copper foils using chemical vapor deposition. After the growth, graphene layers were transfer-printed on $100 \mathrm{~nm} \mathrm{SiO}_{2}$ coated $\mathrm{Si}$ wafers. In the transfer-printing process, a thin layer of photoresist (AZ5214) and an elastomeric stamp were used as a mechanical support for graphene. The details of the process were reported in a previous study. ${ }^{16}$
The highly doped Si substrate works as a gate electrode. The source and drain electrodes were fabricated with standard UV-photolithography process followed by metallization and lift-off steps. The devices were isolated using oxygen plasma etching. The electrical characterizations of the transistors were performed with HP4142B semiconductor parameter analyzer. The rapid thermal annealing of the fabricated transistors was performed by halogen light RTP furnace (RTP-600 S AG Associates/Mini-Pulse). The time trace of the temperature during the RTA process is shown in Fig. 1(b). The transistors were heated up to $300^{\circ} \mathrm{C}$ in $40 \mathrm{~s}$ and kept at $300^{\circ} \mathrm{C}$ for $1 \mathrm{~min}$ and cooled down to room temperature in $5 \mathrm{~min}$, we repeat this process for 10 times. During the RTA process, we run forming gas (5\% hydrogen in nitrogen) into the chamber. We measured transfer and output curves of the devices before and after the RTA process. Fig. 1(c) shows transfer curves of a representative device with silver electrodes before and after RTA. The back-gated graphene transistors show p-type behavior and have minimum conductivity point at a gate voltage of $80 \mathrm{~V}$. We also fabricated top-gated transistors showing ambipolar behavior. To minimize the effects of gate dielectric, we only used back-gated transistors in this study. The transistors have metal contacts without any adhesive layers, like $\mathrm{Ti}$ or $\mathrm{Cr}$. After the RTA, there is around twofold increase in the on current for transistors with $2 \mu \mathrm{m}$ channel length. On-current histogram of the same devices is shown in Fig. 1(d), the improvement in the drain current is significantly larger than the variation of drain current. Raman spectra of graphene before and after RTA process in forming gas are shown in Fig. 1(e), Raman signal does not change significantly with (a)

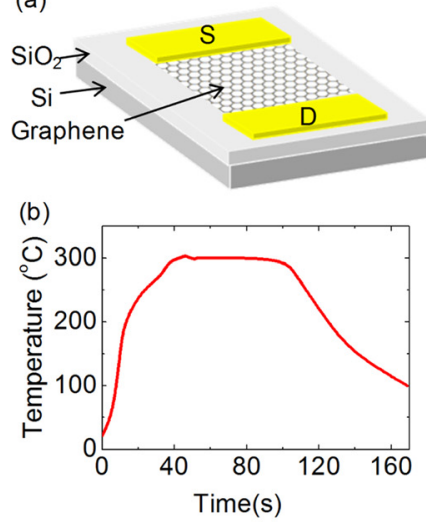

(e)

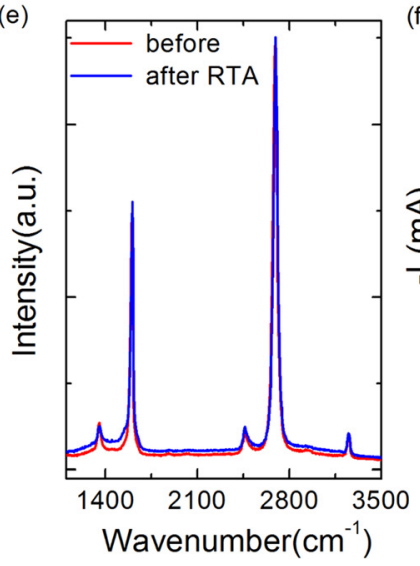

(c)

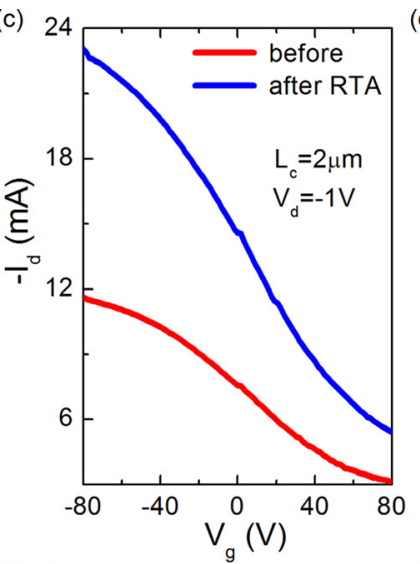

(f)

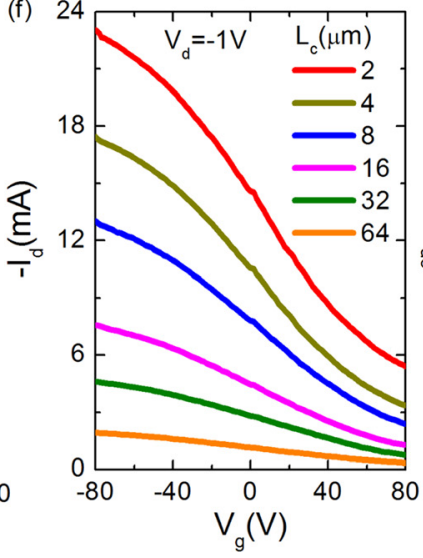

(d)
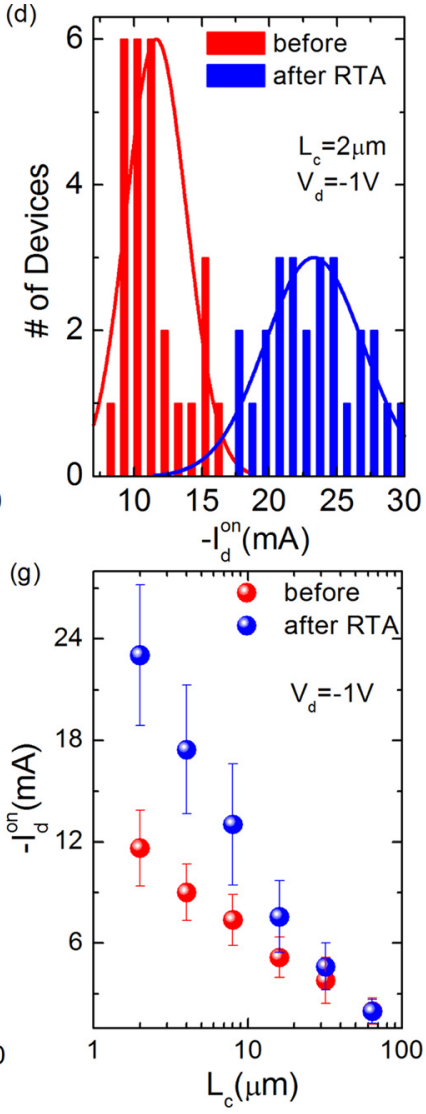

FIG. 1. (a) Schematic drawing of back gated graphene transistors used in this study. (b) Time trace of the temperature of rapid thermal annealing process. (c) Transfer curves of transistors with a channel length of $2 \mu \mathrm{m}$ and a channel width of $100 \mu \mathrm{m}$, before and after the RTA process. These curves are the average of $\sim 30$ devices. The drain voltage is $-1 \mathrm{~V}$ and the transistor uses Ag electrodes. (d) Histogram of drain current before and after the RTA process for devices with $2 \mu \mathrm{m}$ channel length. The improvement in the drain current is significantly larger than the variation of drain current. (e) The Raman spectra of graphene before and after RTA annealing in forming gas $\left(5 \% \mathrm{H}_{2}\right.$ in $\mathrm{N}_{2}$ ) (f) Transfer curves of transistors with various channel lengths between $2 \mu \mathrm{m}$ and $64 \mu \mathrm{m}$. (g) Scaling of drain current as function of the channel length before and after the RTA process. The improvement of drain current decreases with the channel length indicating that the RTA improves the contact resistance. 
RTA annealing in forming gas. The transfer curves for various channel lengths after RTA are given in Fig. 1(f). The scaling of drain current as a function of channel length (Fig. $1(\mathrm{~g})$ ) provides a useful piece of information to understand the effect of RTA. The improvement of drain current decreases with the channel length. This behavior indicates that the RTA reduces the contribution of contact resistance.

We used $\mathrm{TLM}^{7,17}$ to analyze the contact and sheet resistance of the transistors. The total resistance of graphene transistor is a combination of contact resistance, $R_{c}$, and the sheet resistance, $R_{s}$, as $R_{T}=2 R_{c}+R_{s} L_{c} / w$, where $L_{c}$ is the channel length and $w$ is the channel width of the device. The dependence of total resistance on $L_{c}$ provides the contact and sheet resistances of graphene. Fig. 2(a) shows the scaling of the total resistance as a function of channel length before and after the RTA. The scattered plots show the measured total resistance of the devices and the line plots show the linear fitting curves. The channel length varies from $2 \mu \mathrm{m}$ to $64 \mu \mathrm{m}$ and the channel width is $100 \mu \mathrm{m}$. The extracted contact resistance for Ag-graphene contact before and after the RTA is $3.4 \mathrm{k} \Omega \mu \mathrm{m}$ and $1.4 \mathrm{k} \Omega \mu \mathrm{m}$, respectively. We also measured the gate-voltage dependence of the contact resistance from TLM data of more than 100 devices for gate voltages between $-80 \mathrm{~V}$ and $80 \mathrm{~V}$. The extracted contact resistance as a function of gate voltage is shown in Fig. 2(b). Before the RTA, the contact resistance can be modulated
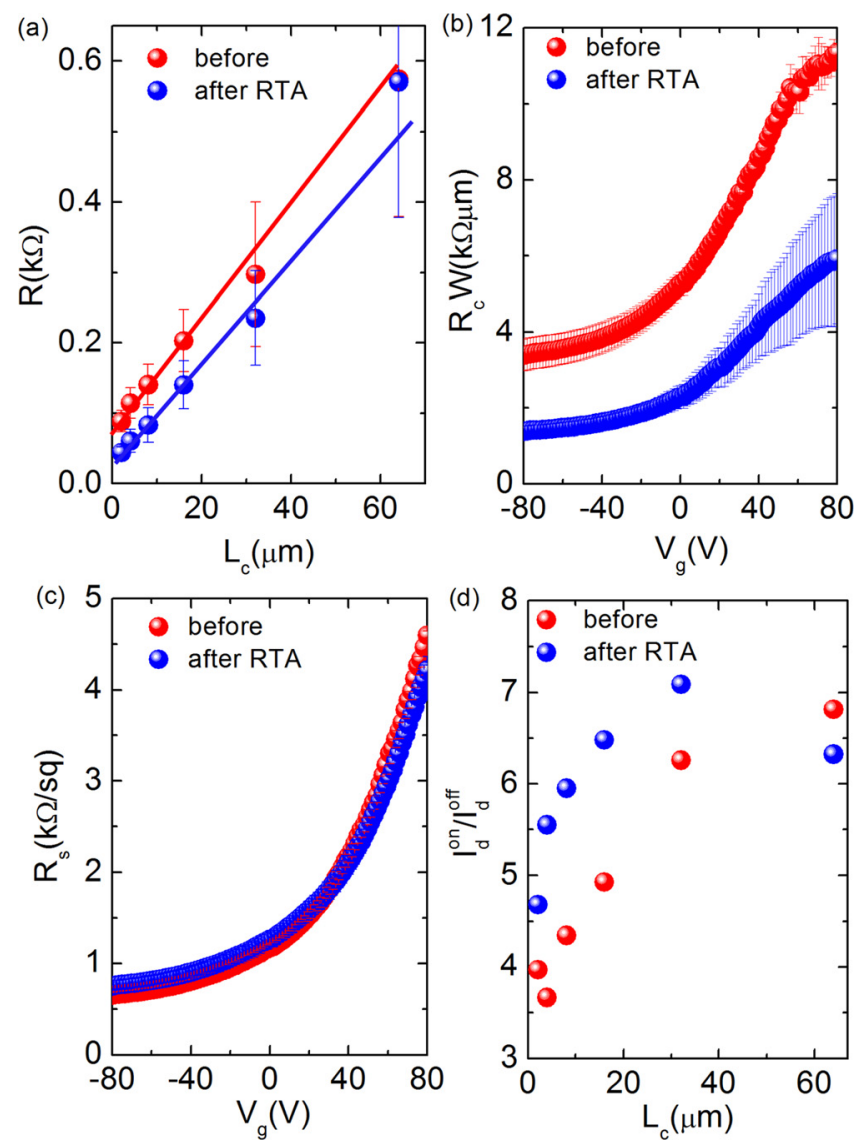

FIG. 2. (a) Scaling of the total resistance of the graphene transistors as a function of channel length, before and after the RTA. The intersection point and the slope provide the contact resistance and the sheet resistance, respectively. Gate voltage dependence of the extracted contact resistance (b), and the sheet resistance (c), before and after the RTA. (d) Scaling of on-off ratio as a function of channel length.
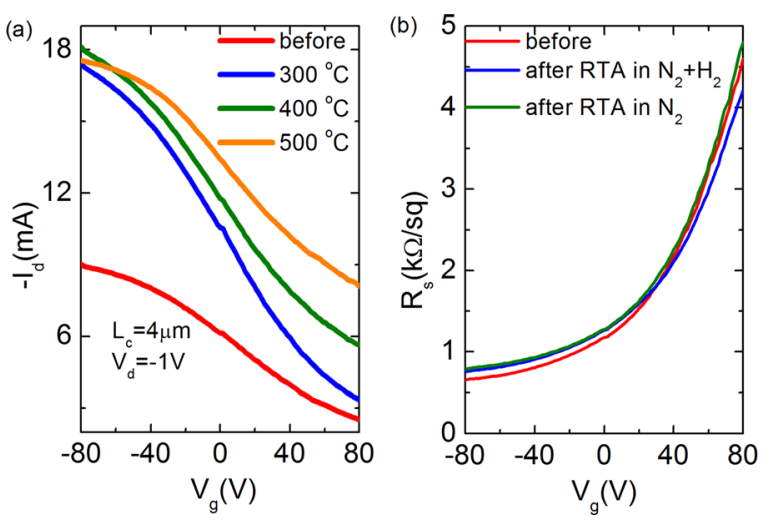

FIG. 3. (a) Effect of the RTA temperature on the tranfer curves of a transistor with a channel length of $4 \mu \mathrm{m}$ and the channel width of $100 \mu \mathrm{m}$. The RTA time is $1 \mathrm{~min}$. (b) The extracted sheet resistance of the graphene before and after RTA process for pure nitrogen gas and forming gas.

from $3.4 \mathrm{k} \Omega \mu \mathrm{m}$ to $11.5 \mathrm{k} \Omega \mu \mathrm{m}$ as we sweep the gate voltage. After the annealing, these values reduced to $1.4 \mathrm{k} \Omega \mu \mathrm{m}$ and $6.0 \mathrm{k} \Omega \mu \mathrm{m}$, respectively. From Fig. 2(b), we can separate the contact resistance into two parts as, $R_{c}=R_{c 0}+R_{c g}\left(V_{g}\right)$, where $R_{c 0}$ is a constant contact resistance which is independent of the gate voltage and $R_{c g}\left(V_{g}\right)$ is the gate modulated contact resistance. The former is a process related resistance which can be reduced by RTA. The latter is a fundamental quantity which depends on the carrier concentration on graphene. We also performed similar analysis for the sheet resistance. The extracted sheet resistance before and after the RTA is given in Fig. 2(c). We did not observe a significant change in the sheet resistance after the annealing process. The sheet resistance is modulated from $750 \Omega / \mathrm{sq}$ to $4.5 \mathrm{k} \Omega /$ sq as we sweep the gate voltage.

The ratio of drain current at "on" and "off" states (so called on-off ratio) is another important operational parameter for graphene transistors. Both contact resistance and sheet resistance are modulated by the gate voltage (see Figs. 2(b) and 2(c)). The on-off ratio of a graphene transistor can be written as $r=\frac{I_{o n}}{I_{\text {off }}}=\frac{R_{c}^{o f f}+L_{L_{C}} R_{s}^{\text {off }}}{R_{c}^{\text {on }}+L_{c} R_{s}^{\text {on }}}$, where superscripts "on" and "off" denote the values at gate voltages of $-80 \mathrm{~V}$ and $80 \mathrm{~V}$, respectively. The modulation of the contact resistance and the sheet resistance by the gate voltage defines the on-off

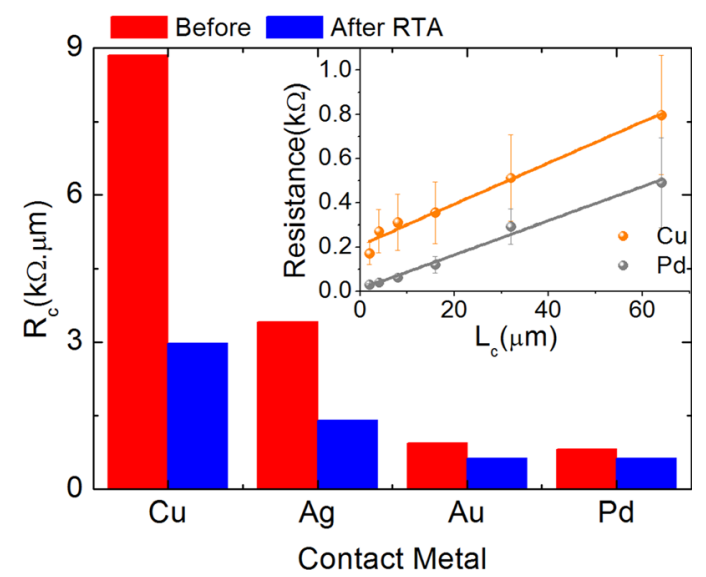

FIG. 4. Extracted contact resistance of graphene for different contact metals; $\mathrm{Cu}, \mathrm{Ag}, \mathrm{Au}$, and $\mathrm{Pd}$. The inset shows the scaling of the total resistance of transistors with a channel width of $100 \mu \mathrm{m}$ for $\mathrm{Cu}$ and Pd contacts. 

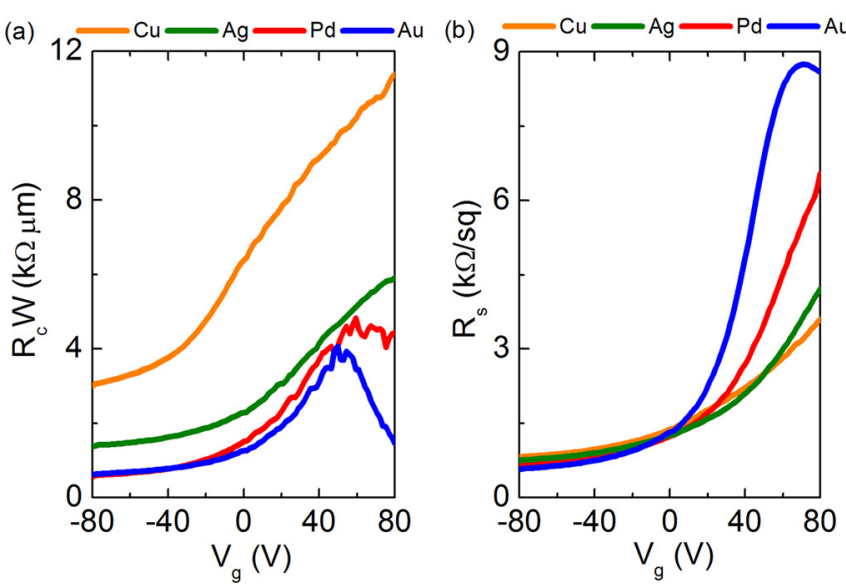

ratio of the devices. Fig. 2(d) shows the scaling of on-off ratio with the channel length. For devices with small channel length $\left(L_{c} \sim 2 \mu \mathrm{m}\right)$, where the transport is limited by the contact resistance, the on-off ratio is defined by the modulation of the contact resistance, $r \approx \frac{R_{c}^{o f f}}{R_{c}^{o n}}=3.6$. For long channels ( $L_{c} \sim 64 \mu \mathrm{m}$ ), the contribution of contact resistance is negligible, and the on-off ratio is characterized by the modulation of the sheet resistance, $r \approx \frac{R_{s}^{o f f}}{R_{s}^{o n}}=7.2$. This trend is clearly seen from the scaling of on-off ratio. Therefore, RTA increases the on-off ratios of transistors with small channel length where the contact resistance is dominant.

In order to find the optimum annealing condition, we performed RTA at various temperatures. Fig. 3 shows the effect of RTA temperature on the transfer curves of a transistor with $4 \mu \mathrm{m}$ channel length. Annealing at $300{ }^{\circ} \mathrm{C}$ provides a significant improvement in the drain current; however, annealing at higher temperatures does not increase the drain current and decreases the modulation of the transistor. These results indicate that RTA at high temperature has detrimental effects on the performance of transistors, likely because of unintentional doping of graphene. We can conclude that RTA at $300^{\circ} \mathrm{C}$ provides the best improvement in the contact resistance. We also studied the annealing atmosphere on the performance of the devices. Fig. 3(b) shows the sheet resistance of graphene before and after RTA annealing in pure nitrogen $\left(\mathrm{N}_{2}\right)$ and forming gas $\left(5 \% \mathrm{H}_{2}\right.$ in $\left.\mathrm{N}_{2}\right)$. Adding hydrogen to annealing atmosphere does not change the sheet resistance of graphene likely due to the short annealing time. Forming gas or nitrogen provides the optimum atmosphere for RTA of graphene transistors.

Fig. 4 shows the change of contact resistance by RTA for various metals; $\mathrm{Cu}, \mathrm{Ag}, \mathrm{Au}$, and Pd. Before RTA, copper has the highest contact resistance of $8.8 \mathrm{k} \Omega \mu \mathrm{m}$. Silver, gold, and palladium have contact resistances of 3.4, 0.94, and $0.82 \mathrm{k} \Omega \mu \mathrm{m}$, respectively. After the RTA process, we observed a significant reduction of contact resistance for copper $(2.9 \mathrm{k} \Omega \mu \mathrm{m})$ and silver $(1.4 \mathrm{k} \Omega \mu \mathrm{m})$. RTA has less profound effect on gold $(0.63 \mathrm{k} \Omega \mu \mathrm{m})$ and palladium $(0.57 \mathrm{k} \Omega \mu \mathrm{m})$ contacts. The inset shows the scaling of total resistance of graphene transistors having copper and palladium electrodes.

To understand more inside about the contacts, we extracted voltage dependence of contact resistance and sheet

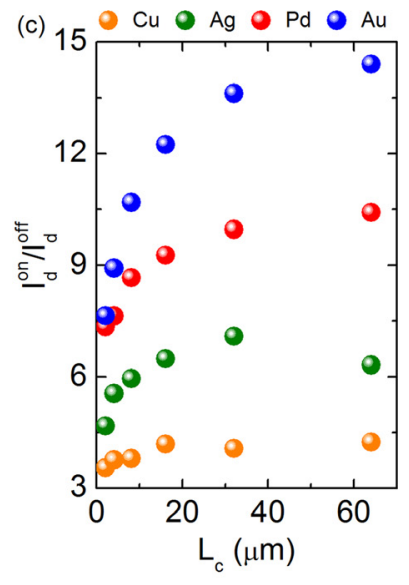

FIG. 5. (a) and (b) Variation of contact resistance $\left(R_{c} W\right)$ and sheet resistance $\left(R_{s}\right)$ with the gate voltage, respectively. (c) Channel length scaling of on-off ratio for various contact metals. resistance for $\mathrm{Cu}, \mathrm{Ag}, \mathrm{Au}$, and Pd. Figs. 5(a) and 5(b) show the extracted resistances of transistors, which use these metals as electrodes. We observe that the contact metal changes the position of the charge neutrality point. This observation can be explained by the contact induced doping in graphene. ${ }^{18}$ Contact resistance and contact induced doping have strong correlation. The contact-induced doping level on graphene is proportional to the contact resistance. Furthermore, the shift of charge neutrality point reduces the observed onoff ratio. Since the range of the gate voltage $(-80 \mathrm{~V}$ to $+80 \mathrm{~V}$ for $100 \mathrm{~nm} \mathrm{SiO}_{2}$ dielectric) is limited by breakdown of the gate dielectric, we do not observe the charge neutrality point for $\mathrm{Cu}, \mathrm{Ag}$, and $\mathrm{Pd}$. The window of the gate voltage limits the observed on-off ratio. Fig. 5(c) shows the calculated on-off ratio as a function of channel length. Au electrodes provide large on-off ratio ( $r=14$ for long channels) due to the low contact resistance and less doping in graphene. On the other hand, $\mathrm{Cu}$ electrodes show small on-off ratio $(r=4$ for long channels) owing to the large contact resistance and associated doping on graphene.

As a conclusion, we studied rapid thermal annealing of graphene transistors to improve contact resistance of graphene-metal junction. We present results of a systematic investigation of device scaling for various RTA conditions and various metal contacts. The results reveal that the contact resistance of graphene-metal junction is a combination of fundamental and process related resistances. The process related contact resistance can be partially eliminated by rapid thermal annealing. We also observed that the contact metals dope the graphene depending on the contact resistance. RTA provides a convenient post-processing technique to reduce contact resistance thus to obtain reproducible device operation.

This work was supported by the Scientific and Technological Research Council of Turkey (TUBITAK) Grant No. 109T209, Marie Curie International Reintegration Grant (IRG) Grant No. 256458, Turkish Academy of Science (TUBA-Gebip).

${ }^{1}$ H. B. Harrison, S. S. Iyer, G. A. Saihalasz, and S. A. Cohen, Appl. Phys. Lett. 51(13), 992 (1987).

${ }^{2}$ Z. Xie and S. P. Murarka, J. Electrochem. Soc. 134(6), C265 (1987).

${ }^{3}$ T. A. Frewen and T. Sinno, Appl. Phys. Lett. 89(19), 191903 (2006).

${ }^{4}$ M. Simardnormandin, IEEE Trans. Electron Devices 32(7), 1354 (1985).

${ }^{5}$ A. Hsu, H. Wang, K. K. Kim, J. Kong, and T. Palacios, IEEE Electron Device Lett. 32(8), 1008 (2011). 
${ }^{6}$ K. N. Parrish and D. Akinwande, Appl. Phys. Lett. 98(18), 183505 (2011). ${ }^{7}$ F. N. Xia, V. Perebeinos, Y. M. Lin, Y. Q. Wu, and P. Avouris, Nature Nanotechnol. 6(3), 179 (2011).

${ }^{8}$ J. A. Robinson, M. LaBella, M. Zhu, M. Hollander, R. Kasarda, Z. Hughes, K. Trumbull, R. Cavalero, and D. Snyder, Appl. Phys. Lett. 98(5), 053103 (2011); T. Mueller, F. Xia, M. Freitag, J. Tsang, and P. Avouris, Phys. Rev. B 79(24), 245430 (2009); J. Knoch, Z. H. Chen, and J. Appenzeller, IEEE Trans. Nanotechnol. 11(3), 513 (2012).

${ }^{9}$ Y. Q. Wu, Y. M. Lin, A. A. Bol, K. A. Jenkins, F. N. Xia, D. B. Farmer, Y. Zhu, and P. Avouris, Nature 472(7341), 74 (2011); Y. M. Lin, A. Valdes-Garcia, S. J. Han, D. B. Farmer, I. Meric, Y. N. Sun, Y. Q. Wu, C. Dimitrakopoulos, A. Grill, P. Avouris, and K. A. Jenkins, Science 332(6035), 1294 (2011); E. Pince and C. Kocabas, Appl. Phys. Lett. 97(17), 173106 (2010); C. Kocabas, H. S. Kim, T. Banks, J. A. Rogers, A. A. Pesetski, J. E. Baumgardner, S. V. Krishnaswamy, and H. Zhang, Proc. Natl. Acad. Sci. U.S.A. 105(5), 1405 (2008).

${ }^{10} \mathrm{~S}$. Datta, Electronic Transport in Mesoscopic Systems (Cambridge University Press, 1995).
${ }^{11}$ K. Nagashio and A. Toriumi, Jpn. J. Appl. Phys., Part 1 50(7), 070108 (2011).

${ }^{12}$ A. W. Tsen, L. Brown, M. P. Levendorf, F. Ghahari, P. Y. Huang, R. W. Havener, C. S. Ruiz-Vargas, D. A. Muller, P. Kim, and J. Park, Science 336(6085), 1143 (2012).

${ }^{13}$ A. D. Franklin, S. J. Han, A. A. Bol, and V. Perebeinos, IEEE Electron Device Lett. 33(1), 17 (2012).

${ }^{14}$ E. Watanabe, A. Conwill, D. Tsuya, and Y. Koide, Diamond Relat. Mater. 24, 171 (2012).

${ }^{15}$ S. M. Song, J. K. Park, O. J. Sul, and B. J. Cho, Nano Lett. 12, 3887-3892 (2012).

${ }^{16}$ O. Salihoglu, S. Balci, and C. Kocabas, Appl. Phys. Lett. 100(21), 213110 (2012).

${ }^{17}$ X. N. Ho, L. N. Ye, S. V. Rotkin, Q. Cao, S. Unarunotai, S. Salamat, M. A. Alam, and J. A. Rogers, Nano Lett. 10(2), 499 (2010).

${ }^{18}$ H. T. Xu, S. Wang, Z. Y. Zhang, Z. X. Wang, H. L. Xu, and L. M. Peng, Appl. Phys. Lett. 100(10), 103501 (2012); G. Giovannetti, P. A. Khomyakov, G. Brocks, V. M. Karpan, J. van den Brink, and P. J. Kelly, Phys. Rev. Lett. 101(2), 026803 (2008). 